\title{
VISUALIZATION OF EXPERIMENTAL DATA IN WEB- BASED VIRTUAL REALITY
}

\author{
Š. Korečko ${ }^{1, a}$, M. Val'a ${ }^{2}$, M. Fekete ${ }^{1}$ \\ ${ }^{1}$ Department of Computers and Informatics, Faculty of Electrical Engineering and \\ Informatics, Technical University of Košice, Slovak Republic \\ ${ }^{2}$ Faculty of Science, Pavol Jozef Šafárik University in Košice, Slovak Republic \\ E-mail: ${ }^{a}$ stefan.korecko@tuke.sk
}

\begin{abstract}
Technological advances in the field of virtual reality and personal computation in general brought us to the era of web-based virtual reality, where virtual environments can be accessed directly from web browsers and without the need of installation of any additional software. Such online virtual environments seem to be a promising tool for scientific data visualization. When accessed through appropriate hardware, such as VR headsets, they also offer full immersion and isolation from external influences. In this paper, we present a prototype solution for a histogram visualization in online virtual environments. The prototype, named NDMVR, has been implemented using A-Frame framework for visualization, React.js for compositionality and JSROOT for histogram data acquisition. Its user interface is primarily adjusted to personal computers and VR headsets.
\end{abstract}

Keywords: virtual reality, extended reality, web-based, data, visualization

Štefan Korečko, Martin Val’a, Martin Fekete

Copyright (C) 2021 for this paper by its authors. Use permitted under Creative Commons License Attribution 4.0 International (CC BY 4.0). 


\section{Introduction and Related Work}

There are several reasons why virtual reality (VR) can be considered a promising tool for scientific data visualization. The first reason is that virtual reality allows us to inspect the data in nontraditional ways. With an appropriate hardware, we can fully immerse into the data and observe them as observing underwater objects when swimming or diving. And the hardware needed for the full immersion is quite affordable. A VR headset costs about 500 Euros and a smartphone can be turned into a headset with a Google Cardboard-like viewer for about 10 Euros. There are also other promising possibilities such as visualization sharing or health improvement. By the visualization sharing we mean that a scientist may invite others to join his or her virtual environment with a particular visualization of a particular data set. Regarding the health, with an appropriate headset a scientist can physically move when browsing through data, instead of just using a mouse or keyboard when sitting in front of a computer. For decades, VR has been connected to the high energy physics research, conducted on particle accelerators, such as the ones at CERN (Geneva) and JINR (Dubna). Via the VENUS (Virtual Environment Navigation in the Underground Sites) project [1], VR had been employed in the development of the LHC (Large Hadron Collider) itself. The infrastructure of LHC in VR is also presented in [2], however with a different purpose. In [2], interactive, didactic, epistemic models and corresponding activities are built for didactic purposes, namely to understand the ALICE (A Large Ion Collider Experiment) detector. The work [2] utilizes the web-based VR, which means that virtual environments can be opened as web pages in a web browser. Another work related to ALICE is [3], which proposes to visualize data acquired in ALICE as 3D trajectories of particles. The authors of [3] focus on compensating a distortion, which occurs when VR headsets are used to display the trajectories.

The development presented here is an original software component named NDMVR (NDiMensional VR). NDMVR allows to visualize two- and three-dimensional histograms (TH2 and TH3) in web-based VR. NDMVR is planned to be primarily used within a larger ecosystem, where dynamic data acquisition will be provided by another original component, called SALSA. Its utilization in other contexts is possible, too. As in the case of [2] and [3], NDMVR contributes to the virtual reality utilization with respect to ALICE, but in a different way. Similarly to [2], it utilizes state of the art technologies for the web-based VR and, as in [3], it focuses on the visualization of data acquired in ALICE. However, [2] visualizes the equipment and not the data and [3] deals with trajectories and not the histograms. The rest of the paper is organized as follows. Section 2 deals with the software architecture of NDMVR and its relation to the data acquisition component SALSA. The current functionality and user interface of NDMVR is described in Section 3 and the paper concludes with remarks on future research and development in Section 4.

\section{NDMVR Architecture}

The NDMVR (N-DiMensional Virtual Rreality) has been designed as a reusable component, utilizing software frameworks A-Frame [4] and React.js [5]. A-Frame is used for 3D visualization and support of various virtual and extended reality hardware and React.js optimizes its performance and allows to make it available in a form of a reusable and customizable component. The current version of NDMVR is available online, at [6], and it runs exclusively on a client side, in a web browser. Its architecture can be seen in Figure 1. The top-level part is $N D M V R$, an npm package that can be incorporated to virtually any web application, created in React.js. NDMVR wraps the VR Scene part, which is responsible for rendering a 3D scene that visualizes histograms of the TH2 or TH3 type. This is done with the help of its two sub-parts, Histogram $3 D$ and VR Camera. Histogram $3 D$ is responsible for the VR scene with the histogram and VR Camera defines the first-person point of view for the user. The data to be visualized as histograms are acquired in the format of the ROOT Data Analysis Framework [7] and NDMVR uses JSROOT [8], an already existing framework for web-based scientific data visualization, to process the data. JSROOT is also used to render a TH1 histogram of a particular bin of the histogram, visualized in the 3D scene. 


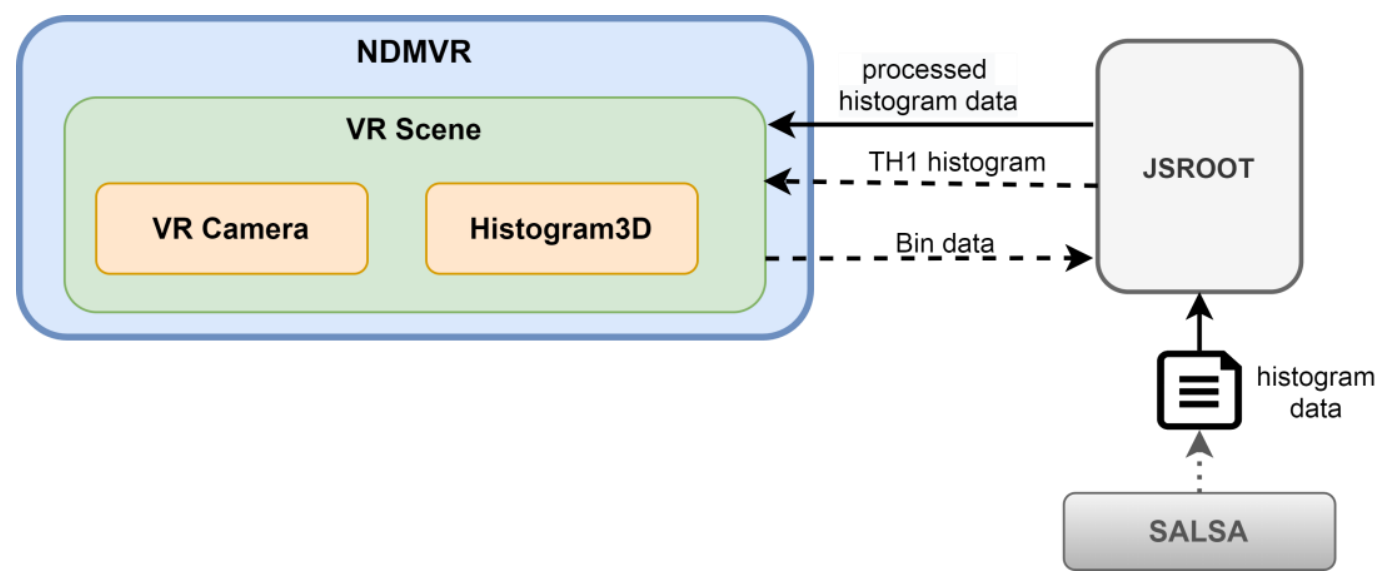

Figure 1. Software Architecture of NDMVR

In the current version of NDMVR, the source of the data is a file. But this will be soon replaced with another original component, named SALSA (Scalable Adaptive Large Structures Analysis) [9], which will provide dynamic data acquisition from multiple data sources simultaneously. SALSA supports distributed network system that can be constructed at a level of clusters, nodes, processes and threads, with arbitrary tree structure. Its user interface is implemented as a web service that can connect to SALSA network and distribute tasks, such as data acquisition, to workers.

\section{NDMVR Functionality and User Interface}

Figure 2 shows how the user interface of NDMVR looks in a web browser when visualizing a two-dimensional histogram. The interface has two display modes, a normal mode and a VR mode.
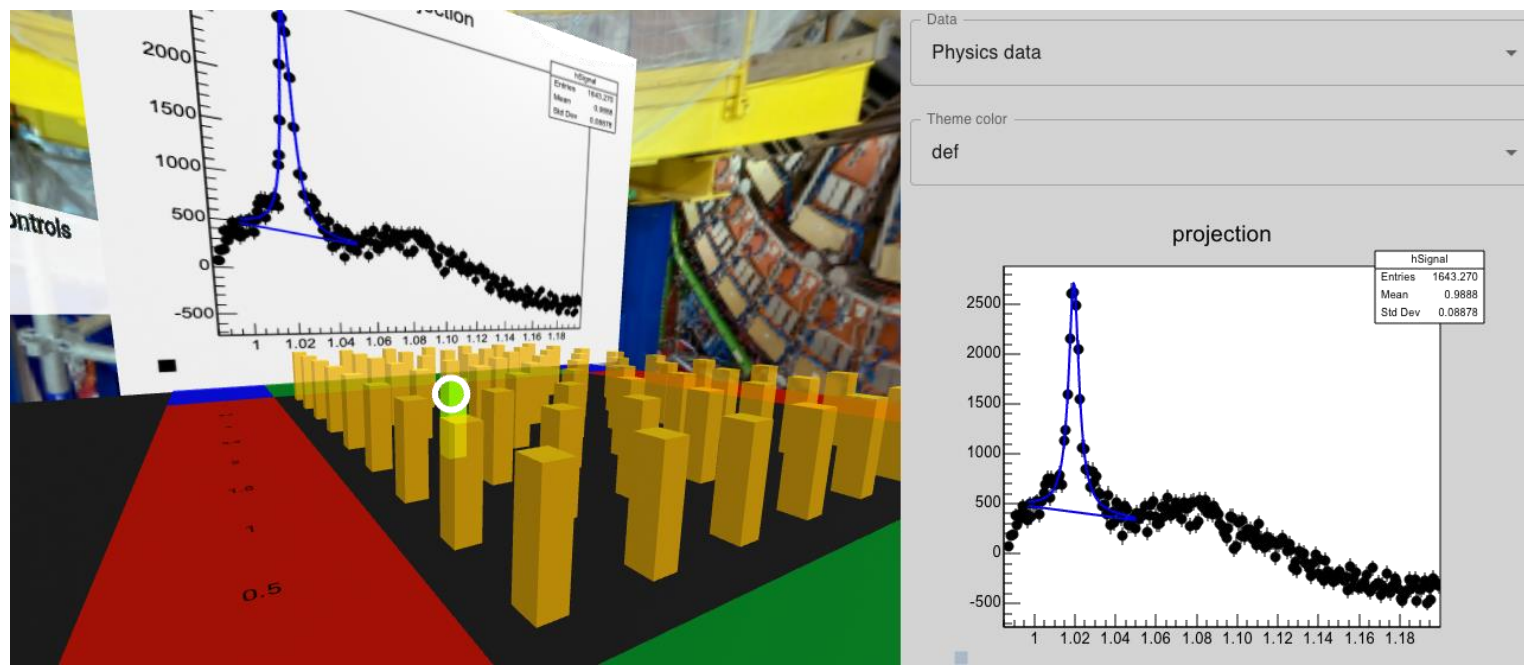

Figure 2. NDMVR user interface with TH2 visualization

The normal mode (Figure 2) is the default one, used when an application using NDMVR is opened in a web browser. The 3D scene with a TH2 histogram is on the left while on the right we can see a TH1 histogram for the selected (green) bin in TH3. The right side also contains settings for the TH1 and the TH1 is also displayed on a 2D plane behind the TH2 in the 3D scene. In the 3D scene, the user can move freely, scroll the histogram up and down along the red and green axis and select a bin and display its TH1. It is also possible to display help panes with detailed instructions on how to use the visualization. Only the 3D scene is shown in the VR mode. Regarding user input, NDMVR is optimized for standard devices (mouse and keyboard) and VR headsets (motion tracking and handheld controllers). 


\section{Conclusion}

The NDMVR component, as presented here, can be regarded a prototype and there is much more that we have in plan. In addition to the dynamic data acquisition via the SALSA component, we also plan several experiments. These will be performed in order to answer related research questions, such as what are the most effective ways of data visualization in VR, whether the assumed benefits of VR will manifest, how easy it is to use this solution and how the data visualization in VR performs on various hardware.

\section{Acknowledgement}

This work has been partially supported by the Slovak Research and Development Agency project APVV-16-0202 and by the grant no. KEGA 035TUKE-4/2019 of the Cultural and Educational Grant Agency of the Slovak Republic, funded by the Ministry of Education, Science, Research and Sport of the Slovak Republic.

\section{References}

[1] Jean-Francis Balaguer, Silvano de Gennaro. VENUS: a virtual reality project at CERN, SIGGRAPH Comput. Graph, Nov. 1996, Vol.30, no.4, pp.40-43. DOI: https://doi.org/10.1145/240806.240812

[2] Christian Klein-Bösing, Philipp Bhatty, Reinhard Schulz-Schaeffer, Stefan Heusler. 3D und Virtual-Reality-Umgebungen zur Vermittlung von Grundlagenforschung am Beispiel des ALICEDetektors am CERN-LHC: Frühjahrstagung der Deutschen Physikalischen Gesellschaft 2020 Bonn, April 2020, ISSN 0420-0195, Available at: https://reposit.hawhamburg.de/handle/20.500.12738/10901 (accessed 25.09.2021)

[3] Julian Myrcha, Tomasz Trzciński, Przemysław Rokita. Virtual reality visualization algorithms for the ALICE high energy physics experiment on the LHC at CERN, Photonics Applications in Astronomy, Communications, Industry, and High Energy Physics Experiments 2017. Vol.10445. International Society for Optics and Photonics, 2017.

[4] A-Frame framework homepage, https://aframe.io/ (accessed 25.09.2021)

[5] React.js homepage, https://reactjs.org/ (accessed 25.09.2021)

[6] NDMVR Demonstrator homepage, https://ndmspc.gitlab.io/ndmvr (accessed 25.09.2021)

[7] ROOT Data Analysis Framework homepage, https://root.cern.ch/ (accessed 25.09.2021)

[8] JSROOT homepage, https://root.cern.ch/js/ (accessed 25.09.2021)

[9] SALSA repository, https://gitlab.com/ndmspc/salsa (accessed 25.09.2021) 